\title{
Catástrofes y nuevos paradigmas del proyecto arquitectónico y urbano
}

\section{Catastrophes and new paradigms of the architectural and urban project}

\author{
${ }^{1}$ Andrea Gritti, ${ }^{2}$ Paola Bracchi \\ ${ }^{1}$ Politecnico di Milano, Milano-Italia \\ 〈andrea.gritti@polimi.it». \\ ${ }^{2}$ Universidad Tecnológica Equinoccial, Quito- \\ Ecuador; Politécnico di Milano, Milano-Italia \\ 〈paola.bracchi@polimi.it〉.
}

Recepción / Received: 30,09, 2017

Aceptación / Accepted: 30, 11, 2017

Publicado / Published: 26, 12, 2017

\begin{abstract}
Resumen:
¿Con cuáles métodos, instrumentos y técnicas la arquitectura y el urbanismo se miden con los efectos de las catástrofes sobre los asentamientos humanos?

En una época que parece caracterizada por la "sociedad del riesgo" y por una "segunda modernidad", parece necesario establecer si esté en curso o menos un cambio paradigmático de la cultura del proyecto arquitectónico y urbano. En esta perspectiva ningún ámbito de acción parece más idóneo de aquél que interpreta las intervenciones conexas con el "ciclo de vida de los desastres" (prevención, respuesta, reconstrucción, mitigación).

A partir del reconocimiento teórico alrededor de los términos del problema (catástrofe, crisis, emergencia, paradigma) la contribución indaga la relación entre proyecto y catástrofe.

La hipótesis es que el proyecto de sistemas complejos, turbulentos y dinámicos tenga que ser sustentado por la integración estratégica entre operaciones de conocimiento del espacio físico, del entorno técnico y del tejido socioeconómico y cultural de los lugares y de las comunidades expuesta al riesgo de manifestación de los desastres.

Para eficacia esta integración tiene que demostrarse abierta a las modificaciones, sea ordinarias o excepcionales, del contexto de referencia.

Metodológicamente se impone la implicación en el proyecto arquitectónico y urbano y a lo largo del ciclo de vida de los desastres, de los factores críticos realzados por mapas, expresados en escenarios y representados, mediante configuraciones adaptables y transitorias.

En perspectiva, el cambio de paradigma anunciado propone el proyecto arquitectónico y urbano, como instrumento esencial en la prevención de los riesgos conexos con la manifestación de las catástrofes.
\end{abstract}

Palabras clave: catástrofe, crisis, emergencia, paradigma, proyecto. 


\begin{abstract}
With which methods, tools and techniques the architecture and the urbanism are connected with the effects of the catastrophes on the human installations?

In an epoch that seems characterized by the affirmation of the "society of the risk" and from a "second modernity", it seems necessary to establish if is in progress or not a paradigmatic change of the culture of the architectural and urban project. In this perspective any field of action appears fitter than the one that interprets the interventions connected with the "cycle of life of the disasters" (prevention, answer, reconstruction, mitigation). Beginning from the theoretical recognition around the terms of the problem (catastrophe, crisis, emergency, paradigm) the contribution investigates the relationship between project and catastrophe. The hypothesis is that the project of complexes, turbulent and dynamic systems, must be considered as the strategic integration among operations of knowledge of the physical space, of the technical environment and of the socio-economic and cultural fabric of the places and the communities exposed to the risk of manifestation of the disasters.

To result effective this integration must be open to the modifications, both ordinary and exceptional, of the context of reference.

Methodologically is necessary to impose in the urban and architectonical project and along the whole cycle of life of the disasters the implication of the critical factors through maps, expressed through scenarios and represented across adaptable and transitory configurations. In perspective the change of announced paradigm aspires to impose the architectural and urban project, as essential tool in the prevention of the risks connected with the demonstration of the catastrophes.
\end{abstract}

Keywords: Catastrophe, Crisis, Emergency, Paradigms, Project.

\section{INTRODUCCIÓN}

Para valorar si está presente un cambio de paradigma en la cultura del proyecto arquitectónico y urbano, ${ }^{1}$ es importante comprender qué significado han asumido en este ámbito términos como crisis, catástrofe, emergencia, resiliencia.

1 Por Proyecto Arquitectónico y Urbano se entiende una visión del proyecto capaz de integrar los aspectos fisico-ambientales con los económicoculturales y tecnológico-ambientales. Se trata de una visión a escalas distintas, entre la del paisaje, la urbana y la arquitectónica para encontrar relaciones estructurales que anclan el proyecto al contexto fisico, cultural y ambiental.
$\mathrm{Su}$ inclusión en el perímetro de las disciplinas proyectuales es en efecto el presupuesto por la superación de teorías y regla, que consideran el proyecto como un sujeto estático, incapaz de medirse eficazmente con lo imprevisible, en general, y con las catástrofes, en particular (Virilio 2007).

Un cambio de paradigma presupone experimentaciones compartidas y un común campo de acción: la repetición de las catástrofes y la intensificación del "ciclo de los desastres" (catástrofe / emergencia / reconstrucción / prevención) constituye de ello una evidente representación. 
Normalmente, el proyecto arquitectónico y urbano pretende contribuir al agotamiento de la fase de emergencia por progresivas adaptaciones del orden anterior a la catástrofe al siguiente, modalidad que pueden demostrarse neutrales o beligerantes en relación con los órdenes originarios. Fenómenos de escala global como los efectos del cambio climático, la limitación de los recursos, la compresión espacio-temporal determinada por la difusión de las tecnologías de la comunicación han transformado la manifestación de las catástrofes en acontecimientos de naturaleza estructural y no episódica, capaz de producir resultados incontrolados (Morini 2014).

Catástrofe tras catástrofe, en la actualidad, se registran: la progresiva extensión temporal de las fases de emergencia; la constante dilación de las intervenciones de reconstrucción; la obstinada inhibición de aquellos de prevención.

Esta tendencia, abundantemente difusa a cada latitud y en cada circunstancia, puede ser invertida solo a través de la introducción de un cambio de paradigma. Se trata de reemplazar a una práctica adicional y parcial, una visión sistémica y global de los fenómenos instalados, basada en la valoración de las invariables en los procesos de modificación. El tipo de proyecto arquitectónico y urbano capaz de interpretar este cambio de condiciones estará basado en la secuencia que unifica mapas, escenarios y configuraciones, pero será diferente del precedente por la expresión de algunas calidades típicamente resilientes: la capacidad de adaptación a nuevas condiciones, la apertura a diferentes principios de ordenación (Desvigne 2008).

Finalmente, esta transformación contesta a listas de peticiones que han sido expresadas por campos del saber colaterales a la arquitectura. La acumulación de ruinas y escombros en los hábitats humanos (Augé 2003) y la asonancia entre la dimensión técnica del proyecto y los procesos destructivos típicos de la economía capitalista (Severino 2003), han sido objeto de agudas observaciones por parte de etnógrafos $\mathrm{y}$ filósofos desde siempre cercanos a las cuestiones propuestas por la arquitectura y preocupados de su progresiva mortificación.

A la desaparición de términos como "prudencia, medida, autocontrol, responsabilidad, solidaridad" de los diccionarios del proyecto corresponde en efecto una cada vez más evidente "voluntad de potencia" respecto a la tierra y la naturaleza, vuelta "inhabitable" en nombre 
de un malentendido progreso técnico (Emery 2011).

En resumen, el cambio de paradigma que en parte se observa y en parte se desea, interpreta muy sencillamente aquella concepción hermenéutica del proyecto que pone al propio centro la "cura" de los lugares y de las comunidades que ellos habitan (Emery 2007).

\section{DESARROLLO DEL ARTÍCULO}

"El futuro es el tiempo que los individuos y la sociedad plantean como posible: pide sabiduría, ética y responsabilidad". (Guidoboni, Mulargia, Teti 2015)

\section{Catástrofe}

Desde un punto de vista etimológico el término Catástrofe deriva del griego Katastrophé y significa trastorno, fin, vuelco. Tiene una connotación negativa que identifica un cambio rápido y en las peores condiciones

físico-espaciales, socioculturales, tecnológico-ambientales, tanto de las personas como de los lugares. ${ }^{2}$

Relativamente al concepto de Catástrofe resulta importante referirse a los estudios de las "teorías de las catástrofes", de Réné Thom, quien describe una catástrofe como un punto crítico que se determina cuando un sistema pierde la su estabilidad. Este tema está desarrollado en el parágrafo relativo al término crisis.
Esta premisa detallada del término permite comprender que hay dos ámbitos fundamentales implicados en el momento en el que ocurre un desastre: de un lado tenemos los aspectos físicos de los lugares, y del otro lado, nos encontramos con los habitantes de estos lugares. El libro Terremoto en Chile, (Das Erdbeben in Chili) de Heinrich von Kleist (1807) representa ambos aspectos previamente descritos. La devastación física de los lugares se une a la devastación espiritual de las víctimas, en una espiral aparentemente sin fin que deja igualmente consternados y aniquilados a los que ofrecen ayuda.

Aunque este género de catástrofe ha existido siempre, hay que considerar que en las últimas décadas el crecimiento demográfico y la expansión de los procesos de urbanización han determinado una mayor exposición y vulnerabilidad de la "sociedad del riesgo" (Beck 1992).

Por lo general, las actuales definiciones de catástrofe elaboran argumentos derivados de las disciplinas científicas, que privilegian aspectos de carácter cuantitativo y se concentran en los efectos posimpacto de un punto de vista sobre todo físico-técnico.

Raramente las disciplinas de la ingeniería, de la geología y de la física toman 
en consideración los aspectos sociales y culturales conexos a un acontecimiento catastrófico, los cuales en el caso de las ciencias humanas son considerados una prioridad, en particular en la siguiente disciplina nueva: la antropología de los desastres (Ligi 2009).

En la antropología de los desastres una catástrofe es considerada

una situación extremadamente crítica que resulta en el momento en el que un agente potencialmente destructivo afecta una determinada población, la cual resulta seguidamente acogida en las nuevas condiciones generadas de vulnerabilidad física y social. (Ligi 2009)

Paralelamente a los daños físicos hay un complejo sistema de problemáticas socioculturales las cuales, a pesar de haber sido generadas bajo las mismas condiciones, pueden tener varios niveles característicamente heterogéneos. La pérdida de la casa, la del lugar de trabajo, de los cariños, o la del propio contexto de referencia en general, es un factor determinante que no puede ser considerado como consecuencia de los daños físicoambientales; al contrario, estos dos aspectos tienen que ser considerados complementarios en el momento en que se interviene en áreas afectadas por acontecimientos catastróficos.

Podríamos entonces afirmar que los instrumentos actuales de intervención, tanto preliminares como en consecuencia de las catástrofes, se focalizan en ámbitos específicos de referencia sin involucrar el conjunto de las transformaciones inducidas sobre el entorno físico, social, cultural, técnico y económico en el que viven las comunidades afectadas.

Si la planificación arquitectónica y urbana se convierte en una disciplina intermitente entre el saber científico y el humanístico, a esta se le pudieran acreditar las competencias de un papel esencial, que se concentraría en la definición de un nuevo paradigma para la interpretación de las acciones necesarias antes, durante y después la manifestación de una catástrofe (Ricci 2012).

Se trata finalmente de ir más allá de las teorías y de la regla del proyecto arquitectónico y urbano, que tienden a producir operaciones estáticas y sectoriales, definitivamente incapaces de llegar a la altura y confrontarse eficazmente con lo imprevisible en general, y con las catástrofes en particular.

\section{Paradigma}

Thomas Kuhn en Las estructuras de las revoluciones cientificas (1962), define un nuevo paradigma como un cambio en las asunciones básicas dentro de una teoría 
científica dominante. En el lenguaje científico el paradigma es definido como un modelo de referencia universalmente aprobado. Término, que deriva del griego Para-Deigma con el significado de ejemplar, ejemplo, pero también como medio de comparación, llega a las ciencias exactas a partir de la esfera filosófica. Platón y Aristóteles lo utilizaban para indicar modelos de referencia universal que permitían comprender y actuar dentro de condiciones específicas.

En la sociología y en la filosofía de las ciencias, paradigma, significa el "complejo de reglas metodológicas, modelos explicativos, criterios de solución de problemas por los cuales se caracteriza una comunidad de científicos en una fase determinada de la evolución histórica de su disciplina" (http://www.treccani.it/vocabolario/paradigm $\mathrm{a} /)$.

Para Kuhn, todas las revoluciones científicas son atribuibles a un cambio de paradigma, o más bien, a un cambio de reglas, de modelos, de métodos y de referencias anteriormente aprobadas que ya no logran contestar las nuevas condiciones.

En Nuovi Paradigmi, Mosé Ricci afirma que:
En el mundo del urbanismo, de la arquitectura y del paisaje un nuevo paradigma es un nuevo modo de trabajar que tiene grandes efectos sobre los espacios de la vida [...] un nuevo paradigma es un modo completamente diferente de fijarse en los espacios del habitar y en su cambio. (Ricci 2012, p. 7)

A diferencia de lo que ocurre en el campo de las ciencias y de la filosofía, en arquitectura la mutación paradigmática, expresa una necesidad que deriva de abajo, va desde la toma de conciencia por parte de las comunidades establecidas a raíz de cambios en el lugar. ${ }^{3}$ El reconocimiento de la intrínseca fragilidad y vulnerabilidad de los asentamientos humanos "frente a los acontecimientos naturales que se transforman en catástrofes" (7) sobresale sin duda del presente que toma lugar entre estos cambios.

\section{Emergencia}

Las intervenciones relacionadas con la interpretación del ciclo de vida de los "desastres" son atribuibles a paradigmas que tienen que ser investigados y eventualmente modificados.

La literatura científica dedicada a este argumento interpreta las relaciones entre las

Mosé Ricci en su ensayo afirma que la necesidad de un cambio de paradigma se advierte en lo que las comunidades expresan. Son las personas que viven en el lugar quienes piden un cambio en lo que es la forma de enfrentarse a las trasformaciones del paisaje. 
acciones de prevención / respuesta / reconstrucción / mitigación de las catástrofes, según una secuencia circular en perpetua renovación, en la cual ningún punto de entrada está unívocamente determinado.

El orden circular de las fases pre, durante $\mathrm{y}$ poscatástrofe, neutraliza los conflictos relativos, tanto a la espera como a la manifestación de la catástrofe misma.

Por este motivo, es necesario volver a considerar la fase de emergencia. Se trata de pasar del estado descrito por la palabra anglosajona emergency, que identifica la tempestividad de acción, a un estado genético-evolutivo de la condición poscatástrofe que identifica el emèrgere de un nuevo orden que establece con el precedente una relación estructural, ya sea desde el punto de vista físico-espacial, que sociocultural y energético-ambiental.

Las investigaciones relativas a las propiedades físicas, matemáticas, biológicas, filosóficas (emergentismo) sociológicas de los sistemas complejos y su arquitectura hacen referencia al término latino emérgere. Tales investigaciones parten del presupuesto que un conjunto, una colectividad, es más que la suma de sus partes, ya que presenta propiedades emergentes no identificables en las componentes individuales. ${ }^{4}$

En primera instancia, la emergencia puede ser considerada el proceso de formación de sistemas más complejos $\mathrm{y}$ turbulentos con respecto de los iniciales. Las propiedades emergentes son, por lo tanto, imprevisibles e inesperadas, no pueden ser previstas con base al estudio de los elementos constitutivos individuales, sino solo por medio de la descripción y la interpretación de las relaciones $y$ de las interacciones estructurales entre las partes. Esta actividad predictiva se solapa con la dimensión proyectual.

Por tanto, bajo el punto de vista de los órdenes físico-espaciales, socioculturales $\mathrm{y}$ técnico-económicos, un sistema complejo resulta emergente, si la transformación de sus componentes originales determina la constitución de nuevos principios de orden, sobre la base de procesos, que interrogan la morfología ${ }^{5}$ como "ley de estructura de los

\footnotetext{
4 Se hace referencias a las investigaciones sobre la "teoría de la complexidad", desde los primeros estudios de Henri Poincaré al final del siglo XIX, para llegar a los estudios de Ilya Prigogine en termodinámica en los sesenta del siglo XX, y a los estudios socio-filosóficos de Edgar Morin sobre la racionalización del pensamiento complejo.

5 Morfología (del griego morphé "forma" y lògos "discurso"), en arquitectura se entiende como los estudios de las formas del espacio construido, abierto y de relaciones. El concepto de forma puede ser analizado de distintas maneras, la forma puede ser vista no solo como figuras
} 
sistemas residenciales dinámicos" (Crotti 1991, p. 37).

La estructura de las relaciones que establece la necesaria y recíproca posición de las partes dentro de la totalidad es el resultado de un proceso de transición de condiciones desde el estado de inestabilidad al de estabilidad que presupone la progresiva subordinación de las partes al todo. (Crotti 1991, p. 38)

Estos procesos asumen una precisa connotación morfo-genética, por la que cada componente deriva de la desestabilización "catastrófica" y repentina de una forma previa.

En una segunda instancia, el término emergencia se refiere a una circunstancia imprevista, a un momento crítico que induce un cambio inesperado de los órdenes consolidados.

\section{Crisis, resiliencia y morfo-génesis}

El término crisis, que deriva del griego Krisis, derivado de Krino (separar distinguir, juzgar), significa literalmente "momento que separa una manera de ser o una serie de fenómenos de otro diferente".

Crisis, emergencias, catástrofes son por lo tanto acontecimientos que determinan una discontinuidad, una rotura, un cambio de

exteriores sino también como estructura. En este último caso se mira a los que pueden ser las posibles relaciones entre formas espaciales distinta para detectar las relaciones entre ellas y las estructuras formales que la determinan. órdenes previamente organizado y señalan el paso de una condición de estabilidad a una de inestabilidad, en el que formas reconocibles se hacen disponibles a tanto a órdenes nuevos como transformados, atados a los precedentes de modo generativo.

La rotura de una conformación existente, a favor de otra diferente, con la cual la primera mantiene relaciones estructurales, es una constante de las teorías genéticas: esta se encuentra en los estudios de Ilya Prigogine (1940), dedicado a la irreversibilidad de los sistemas termodinámicos y en aquellos de René Thom (1965-1972), consagrado a la morfogénesis.

En los modelos morfo-genéticos, discontinuidad y continuidad pueden coexistir dentro del mismo proceso trasformativo. Thom afirma que:

$$
\begin{aligned}
& \text { es posible constatar variaciones } \\
& \text { discontinuas, o bien pequeñas } \\
& \text { alteraciones sistémicas, que, a su vez, } \\
& \text { determinan sustanciales mutaciones } \\
& \text { morfológicas, en el momento en el que se } \\
& \text { manifiesta aquel estadio crítico que se } \\
& \text { denomina 'catástrofe"' (Thom 1972). } \\
& \text { La catástrofe determina una } \\
& \text { discontinuidad, es decir, un cambio profundo } \\
& \text { del sistema existente y la definición de un } \\
& \text { nuevo orden de relaciones que no tiene con el } \\
& \text { precedente, sino más bien puntos parciales de } \\
& \text { contacto, que dependen de su estado original. }
\end{aligned}
$$


Los órdenes permanentes, constituyen por lo tanto la estructura profunda del sistema al cual se refieren constantemente las variaciones morfológicas. En el lenguaje de las formas del habitar, emergencias $\mathrm{y}$ catástrofes, como acontecimientos imprevistos, determinan discontinuidad, en virtud de la cual se manifiesta la "materia originaria" (Martí Arís 1993): la esencia de los lugares, sus fundamentos culturales y sus dinámicas sociales. En definitiva, el nuevo paradigma de la emergencia por causa de catástrofes concierne, por una parte, el logro de una condición estable a partir de un desorden inicial en el interior de sistemas complejos; por otra parte, tiene que ver con la capacidad del mismo sistema de transformarse sin perder sus caracteres fundamentales y originarios.

Desde este punto de vista, la estabilidad y la inestabilidad de un sistema complejo no tienen que ser considerados términos opuestos, si no complementarios, ya que "las catástrofes son los puntos de discontinuidad en los procesos de evolución de las formas" (Terpolilli 2005, p. 10) que hacen emerger varias condiciones originarias, tanto como los estados de límite.

En términos operativos y de proyecto, cabe destacar que los tiempos y los espacios de la emergencia tras catástrofes se amplían cada vez más, y su respectiva asiduidad requiere atención constante. Por lo tanto, se imponen Nuevos Paradigmas con el objetivo de superar las lógicas del socorro improvisado e interpretar crisis, catástrofes y emergencias como variables de los procesos proyectuales bajo los cuales se lleva a cabo la transformación local.

Un paso fundamental en esta dirección consiste en considerar el proyecto arquitectónico y urbano como un proceso, abierto no solo a las exigencias de la modificación, sino también a las determinadas condiciones de la incumbencia de los riesgos y del reconocimiento de las vulnerabilidades. De esta manera parece ser evidente como las disciplinas del proyecto tengan que madurar una específica correlación con el concepto de resiliencia. En el contexto de las ciencias duras el término resiliencia indica la propiedad de algunos sistemas de conservar la misma estructura o de cobrar de nuevo la forma originaria resultado de un proceso de deformación.

Por asociación el concepto de resiliencia indica en el campo de la arquitectura y el urbanismo la capacidad de un sistema de vida o de una estructura espacial de adaptarse a los cambios impuestos a los contextos afectados por fenómenos y procesos de particular fuerza $y$ 
amplitud. Desde este punto de vista la resiliencia no es solo una forma de resistencia frente a la amenaza de roturas dramáticas y tampoco, al revés, una renovación de la destrucción creadora a la Schumpeter, sino un arte de vencer en una manera muy distinta que el Occidente, acostumbrado a construir límites intransitables para contrastar sus amenazas, está descubriendo (Nicolin, 2014, p. 53).

Según Pierluigi Nicolin (2014), la aplicación de estos conceptos a las disciplinas arquitectónicas y urbanas permite volcar la expresión latina frangar, no flectar (me romperé, pero no me doblaré), en el más consciente flectar, no frangar (me doblaré, pero no me romperé). Aceptando la implicación, o bien aferrándose a la misma con el objetivo de adherirse a las razones más profundas del propio contexto, el proyecto arquitectónico y urbano, puede resultar ser resiliente. La explotación de las mismas propiedades plásticas, en esta perspectiva no sigue el objetivo de restablecer el estadio inicial del sistema, sino de "encontrar soluciones abiertas" y de explorar "universos narrativos incompletos" (Nicolin 2014, p. $53)$.

\section{Mapas, escenarios y configuraciones transitorias}

Finalmente, el deseado cambio de paradigma consiste en la asimilación de los conceptos de crisis y catástrofes y de las prácticas de emergencias conectadas en el proceso del proyecto para reemplazar una visión parcial con una global de los fenómenos del habitar, con la habilidad de interpretar y valorizar la dialéctica entre los aspectos de permanencia y variabilidad.

Esta operación impone una consolidación de las capacidades de análisis retrospectivas y de valorización pre-dictiva del proyecto arquitectónico y urbano. Por una parte, se tienen que describir e interpretar críticamente las condiciones de los contextos anteriores a las catástrofes y, por otra parte, se tienen que prever los posibles escenarios que pudieran ocurrir después de su manifestación: en ambos casos renovando la estructura metodológica del proyecto, o bien recurriendo a formas compartidas de narración, adjuntas a la colección de indicadores verificables.

En esta perspectiva se asume un particular relieve de la determinación de las configuraciones transitorias: aquellas conformadas con las estructuras relacionables emergentes o aquéllas ligadas a las preexistentes y reconocidas como fundamentales "naturalezas intermedias" 
(Desvigne 2012) constantemente disponibles a la variación.

Bajo el perfil metodológico se pueden describir las siguientes tres operaciones: 1. La creación de mapas, tanto de síntesis como de detalle, de las condiciones anteriores y resultantes de la manifestación de las catástrofes; 2. La definición de escenarios, narrada con los instrumentos proyectuales y capaz de identificar estructuras y sistemas de relación sobre los que apoyarse para la individuación de tácticas y estrategias resilientes; 3. La determinación de configuraciones coherentes con las condiciones emergentes y con aquellas originarias.

La innovación paradigmática consiste en reconocer cómo el proyecto arquitectónico y urbano, no se refiere solo a la tercera de las fases previamente descritas, si no se realiza contemporáneamente en presencia de las otras dos, se debe poder retomar para valorar la coherencia del proceso, y en el caso que resulte necesario, revelar y corregir las debilidades de este último.

Con el uso de los mapas se podrán describir e interpretar sistemas y estructuras en escenarios de los acontecimientos catastróficos. Se usarán con las siguientes aplicaciones: la localización de las lagunas, las fracturas $y$ las discontinuidades determinadas por los desastres, pero también con el objetivo de notar los elementos permanentes, las señas y las huellas que no han sido de-estabilizadas por los acontecimientos catastróficos. Una versión especial de los mapas será dedicada al análisis puntual de los daños y de los impactos (con un tipo específico haciendo referencia a la producción de escombros), puestos en correlación con la geografía del abandono anterior al acontecimiento catastrófico. Dinámicamente concebidos, con respecto al espacio, al tiempo y a los temas, estos mapas tienen que configurarse como el principal soporte en la interpretación del ciclo de vida de los "desastres".

A los escenarios se les tiene que confiar la elaboración de relaciones entre "lo que queda" (Benjamín, 1931 y Agamben, 2000) y "lo que será". La interacción entre estas dos condiciones de las comunidades instaladas puede determinar relaciones inéditas sobre las cuales radicar la dimensión narrativa de nuevos espacios y nuevos tiempos.

Las configuraciones son experimentaciones dedicadas a las intervenciones en fase de emergencia, reconstrucción y prevención, que se miden con las complejidades de los contextos indagados, articulando las contribuciones 
ofrecidas por medio de reconocimientos de mapas y de las narraciones usando escenarios, de las catástrofes precedidas.

Ellas tienen que ser ancladas a la estructura profunda de los asentamientos y al mismo tiempo garantizar la transformación, en vista de la inevitable renovación de la espiral inducida por el ciclo de vida de los desastres.

Los proyectos concebidos gracias a la aplicación de este renovado paradigma elaboran una serie de variaciones de principios unitarios: por medio de este, se miden las condiciones anteriores, mientras que mediante las otras, aquellos determinados por la manifestación de los acontecimientos catastróficos.

Esta metodología es auténticamente y conscientemente participativa, ofrece a las comunidades resilientes la posibilidad de intervenir en todas las fases del proceso: elaboración de mapas, definición de las narraciones, promoción de nuevas configuraciones.

En esta perspectiva, los contextos dónde se agrupan los efectos de una catástrofe, representan el campo de prueba para la realización de experimentos compartidos dedicados a consolidar $y$ formalizar el anunciado cambio de paradigma.

\section{RESULTADOS Y DISCUSIÓN}

La metodología previamente descrita constituye un cambio profundo de los métodos, de los instrumentos y de las técnicas del proyecto arquitectónico urbano. La principal transformación consiste en la adopción de un enfoque, paso a paso, que llega a la arquitectura y al urbanismo desde las disciplinas del medio ambiente. El objetivo a alcanzar es la determinación de estructuras capaces de afrontar de modo sistémico los efectos de una catástrofe o de preparar las comunidades instaladas a su manifestación, no solo durante la emergencia, durante todas las fases del ciclo de los desastres.

\section{CONCLUSIONES Y RECOMENDACIONES}

Las catástrofes representan la ocasión para poner a prueba la obsolescencia de los instrumentos proyectivos que deberían acompañar la evolución de la "sociedad del riesgo" (Beck 1992).

El objetivo es innovar métodos, instrumentos y técnicas del proyecto de arquitectura para que los conceptos de deformación, flexibilidad y adaptabilidad 
sean proporcionados a las necesidades de las comunidades golpeadas por los acontecimientos catastróficos. En esta perspectiva el proyecto arquitectónico $\mathrm{y}$ urbano es llamado a "preparar el campo". Para el futuro en tres pasos fundamentales:

1. La definición de estructuras y reglas esenciales;

2. La programática renuncia al control sobre cada individual elemento;

3. El descifre de los órdenes posibles.

Este último paso no puede ser cumplido sin la participación de las comunidades instaladas al desarrollo proyectivo. A ellas, después de una catástrofe, corresponde en vía exclusiva, la tarea de invertir la relación entre pasado y futuro, entre memoria y esperanza.

En los lugares devastados por los desastres, ninguna narración proyectiva $\mathrm{y}$ ningún escenario operativo es legítimo $\mathrm{y}$ posible sin la implicación de los que saben medir el "tiempo que queda" (Agamben 2000).

\section{REFERENCIAS BIBLIOGRÁFICAS}

\section{Libros}

Agamben, G. (2000). Il tempo che resta. Un

commento alla Lettera ai Romani. Turín:

Bollati Boringhieri.

Augé, M. (2003). Le temps en ruines.
París: Galilée.

Beck, U. (1992). Risk Society. Towards a

New Modernity. Los Ángeles: Sage

Publishing.

Desvigne, M. (2009). Intermediate

Natures. The landscape of Michel Desvigne.

Berlín: Birkhauser.

Emeryi, N. (2011). Distruzione e progetto.

L’architettura promessa. Milán: Marinotti.

Emeryi, N. (2007). Progettare, costruire,

curare. Per una deontología

dell'architettura. Bellinzona:

Casagrande editore.

Guidoboni, E.; Mulargia, F., y Teti, V. (2015). Prevedibile/imprevedibile.

Eventi estremi nel prossimo futuro. Italy: Rubbettino editore.

Johnson, S. (2001). Emergence: The

Connected Lives of Ants, Brains, Cities, and

Software. New York: Scribner.

Kuhn, Thomas S. (1962). The structure of

scientific revolutions. Chicago: University of Chicago Press.

Ligi, G. (2009). Antropologia dei disastri.

Bari: Laterza

Morini, S. (2014). Il rischio. Da Pascal a

Fukushima. Turín: Bollati Boringhieri.

Ricci, M. (2012). New Paradigms. Trento:

LISt editor.

Severino, E. (2003). Tecnica e Architettura.

Milán: Raffaello Cortina editore. 
Storchi, S., y Toppetti, F. (2013). Terremoto Emilia: le forme della ricostruzione. Firenze: Alinea.

Tagliabue F., y, Bassoli N. (2016). Stem

Procedure. Strategie di rigenerazione post sisma. Sant'Argangelo di Romagna, Italia: Maggioli editore.

Virilio, P. (2007). L'Université du desastre. París: Edition Galilée.

Waldheim, C. (2000). The landscape urbanism reader. Princeton: Princeton architectural press.

\section{Capítulo en un libro}

Benjamín, W. (1931). "Der destruktive Charakter". En Tiedemann, R. y H. Schweppenhäuser (eds.). Gesammelte Schriften. Frankfurt am Main, pp. 396401.

Crotti, S. (1991). "Determinazioni progettuali della morfogenesi urbana”. En D'Alfonso, E., y E. Franzini (eds.). Milán: Guerini. Terpolilli, C. (2005). "Temporaneo e transitorio nell'architettura contemporánea". En R. Bologna y C. Terpolilli (eds). Emergenza del progetto. Progetto dell'emergenza. Architetture contemporaneità. Milán: Federico Motta. Von Kleist, H. (1810). Das Erdbeben in Chili. En E. von Kleist. (1810). Berlín: Rweimer.

\section{Articulos en revistas}

